\title{
Primeiro registro de Guyalna cuta em pinhão- manso no Estado de Mato Grosso do Sul
}

\author{
Harley Nonato de Oliveira ( $\left.{ }^{*}\right)$; Nilza Maria Martinelli (2); Patrícia Paula Bellon (1,3); Douglas \\ Henrique Bottura Maccagnan ( $\left.{ }^{4}\right)$ \\ (') Embrapa Agropecuária Oeste, Caixa Postal 449, 79804-970 Dourados (MS), Brasil. \\ (2) Universidade Estadual Paulista Júlio de Mesquita Filho, Departamento de Fitossanidade, 01049-010 Jaboticabal (SP), Brasil. \\ (3) Universidade Federal da Grande Dourados, Caixa Postal 533, 79804-970 Dourados (MS), Brasil. \\ (4) Universidade Estadual de Goiás, Unidade Universitária de Iporá, 76200-000 Iporá (GO), Brasil. \\ (*) Autor correspondente: harley@cpao.embrapa.br; harley.oliveira@embrapa.br
}

Recebido: 4/nov./2011; Aceito: 19/jul./2012

\begin{abstract}
Resumo
Trata-se do primeiro registro de ocorrência de Guyalna cuta (Walker) (Hemiptera: Cicadidae) em pinhão-manso, Jatropha curcas L. (Euphorbiaceae) no Estado de Mato Grosso do Sul. Adultos desse inseto foram observados na parte aérea da planta, durante novembro e dezembro de 2008 e de 2009. Em Mato Grosso do Sul, existem plantios comerciais e experimentais do pinhão-manso, tornando-se necessários estudos relacionados a este inseto, a fim de prevenir eventuais danos à cultura.

Palavras-chave: Hemiptera, Cicadidae, cigarra, Jatropha curcas, ocorrência.
\end{abstract}

\section{First record of Guyalna cuta on physic nut in Mato Grosso do Sul State}

\section{Abstract}

This is the first record of the occurrence of Guyalna cuta (Walker) (Hemiptera: Cicadidae) in physic nut plants, Jatropha curcas L. (Euphorbiaceae) in the State of Mato Grosso do Sul, county of Dourados. Adults of this insect were observed in the plant canopy during the months of November and December in 2008 and 2009. As there are commercial and experimental plantations of physic nut in Mato Grosso do Sul, further studies about this insect are necessary for preventing eventual damage to crop production.

Key words: Hemiptera, Cicadidae, cicada, Jatropha curcas, occurrence.

As cigarras são insetos comumente observados alimentando-se de seiva de plantas em ambientes silvestres (Martinelli e Zucchi, 1997a). Contudo, com a supressão de seus hospedeiros nativos, esses insetos começaram a adaptar seu hábito alimentar, passando a causar danos econômicos de forma generalizada em plantaçóes de espécies cultivadas, tornando-se muitas vezes pragas, como ocorre para a cultura do café e da espécie florestal paricá (Martinelli e ZuCCHi, 1997b; Zanuncio et al., 2004).

Atualmente no Brasil estão registradas 156 espécies de cigarras (SANBORN, 2008; SANTOS e MARTINELli, 2009a,b; SAntos et al., 2010; Maccagnan et al., 2011; SAnborn, 2011a; Boulard e Martinelli, 2011). No entanto, esse número pode estar subestimado, visto que os diferentes biomas existentes no país proporcionam condições para grande diversidade de espécies de cigarras (SANBORN et al., 2011). Para o Estado do Mato Grosso do Sul estão registradas apenas as espécies Quesada gigas (Olivier), Fidicina mannifera (Fabr.), Dorisiana viridis (Olivier) e D. drewseni (Stål) (Aoki et al., 2010). A falta de estudos a respeito desse grupo de insetos se reflete na pouca informação disponível sobre sua biologia e interaçóes ecológicas como a distribuição e plantas hospedeiras relacionadas, entre outras.

No município de Dourados, Mato Grosso do Sul, em novembro e dezembro de 2008 e de 2009 , verificou-se ocorrência de cigarras em uma área de 1,75 ha cultivada com a cultura do pinhão-manso (Jatropha curcas L.), sendo a área total de plantio, de aproximadamente 12 ha. Adultos desse inseto foram observados na parte aérea das plantas do pinhão-manso; também foram observados orifícios circulares no solo, em torno das plantas, que são indicativos da saída das ninfas das cigarras. Tais orifícios demonstram que estas ninfas estavam atacando as raízes das plantas, o que pode explicar a clorose geral das folhas do pinhão-manso, sintoma similar ao causado em plantas de café (RiBEIRo et al., 2006).

Adultos de cigarras foram coletados com auxílio de 32 armadilhas adesivas de coloração amarela $(24,5$ $\mathrm{cm}$ de altura x $10 \mathrm{~cm}$ de largura), instaladas na área em novembro e dezembro de 2008 e 2009. Amostras dos espécimes coletados estão depositados na Coleção Entomológica do Departamento de Fitossanidade da Faculdade de Ciências Agrárias e Veterinárias 
da UNESP (FCAV/UNESP), Jaboticabal (SP), e na Coleção Entomológica da Embrapa Agropecuária Oeste, Dourados (MS).

Os insetos foram identificados como Guyalna cuta (Walker) (Hemiptera: Cicadidae), espécie pequena e de asas completamente hialinas. O gênero Guyalna (Fidicinini: Guyalnina) foi determinado por Boulard e Martinelli (1996), separando-o do gênero Fidicina por possuir três artículos nos tarsos, timbacalipto lobulado e arredondado, cabeça tão larga ou ligeiramente mais larga que o mesonoto e olhos pouco proeminentes.

A cigarra G. cuta já havia sido relatada para o Brasil, porém sem ser feita referência mais específica sobre a localidade (Metcalf, 1963). A ocorrência de G. cuta também foi constatada para a Guiana Francesa e para a Argentina (Metcalf, 1963; Boulard, 1999; Sanborn, 2011b). Além desses registros de ocorrência, as únicas informaçôes disponíveis na literatura são referentes à descrição do som emitido pelo macho (Boulard, 1999) e sobre os parâmetros térmicos apresentados pela espécie (SANBORN et al., 2011).

Em novembro e dezembro de 2008 foram coletadas, respectivamente, 114 e seis $G$. cuta, representando a média de 3,6 e 0,2 cigarras por armadilha. Esses valores indicaram que $90,6 \%$ e $8,7 \%$ das armadilhas capturaram essa cigarra. No mesmo período em 2009, coletou-se o total de 15 e nove $G$. cuta, respectivamente, onde foi verificada a presença média de 0,46 e 0,28 cigarras por armadilha. Desse total, $37,5 \%$ e $25 \%$ das armadilhas instaladas possuíam o inseto.

Em vista do primeiro registro de G. cuta em Dourados (MS), e o possível potencial de dano que esse inseto pode causar em J. curcas, recomenda-se a realização de estudos relacionados à sua biologia, ao comportamento, à distribuição geográfica do inseto, bem como produtos que podem ser utilizados para seu controle.

\section{AGRADECIMENTOS}

Os autores agradecem à Fundação de Apoio ao Desenvolvimento do Ensino, Ciência e Tecnologia do Estado de Mato Grosso do Sul (FUNDECT) e à Financiadora de Estudos e Projetos (FINEP), pelo apoio financeiro.

\section{REFERÊNCIAS}

AOKI, C.; LOPES, F.S.; SOUZA, F.L. Insecta, Hemiptera, Cicadidae, Quesada gigas (Oliver, 1970), Fidicina mannifera (Fabricius, 1803), Dorisiana viridis (Oliver, 1970) and Dorisiana drewseni (Stal, 1954): first records for the state of Mato Grosso do Sul, Brasil. Check List: Journal of species lists and distribution, v.6, p.162-163, 2010.

BOULARD, M. Postures de cymbalisations et cartes d'identité acoustique des Cigales. 3. Espéces tropicales dês savanes et milieux ouverts (Cicadoidea, Cicadidaes et Tibicinidae). Ephe Biologie et Evolution des Insectes, v.11/12, p.77-117, 1999.
BOULARD, M.; MARTINELLI, N.M. Révision des Fidicinini. Première partie: Sous tribo nouvelle des Fidicinina (Cicadomorpha, Cicadoidea, Cicadidae). Ephe Biologie et Évolution des Insectes, v.9, p.11-81, 1996.

BOULARD, M.; MARTINELLI, N.M. Nouvelles cigales néotropicales de las sous-tribu des Guyalnina (Rhynchota, Cicadidae, Cicadinae, Fidicinini). Lambillionea, v.111, p.219-232, 2011.

MACCAGNAN, D.H.B.; MARTINELLI, N.M.; GOEMANS, G.; GUILHERME, E. Zammara smaragdula Walker, 1858 (Hemiptera: Cicadidae): First record for Brazil. Check List, v.7, p.563-564, 2011.

MARTINELLI, N.M.; ZUCCHI, R.A. Primeiros registros de plantas hospedeiras de Fidicina mannifera, Quesada gigas e Dorisiana drewseni (Hemiptera: Cicadidae). Revista de Agricultura, v.72, p.271-281, 1997a.

MARTINELLI, N.M.;ZUCCHI, R.A. Cigarras (Hemiptera, Cicadidae, Tibicinidae): distribuiçáo, hospedeiros e chave para as espécies. Anais da Sociedade Entomológica do Brasil, v.26, p.133-141, $1997 \mathrm{~b}$.

METCALF, Z.P. General catalogue of the Homoptera: fascile VIII. Cicadoidea. Part 1: Cicadidae. Raleigh: Waverly Press, 1963. 492p.

RIBEIRO, R.; PEREIRA, M.F.A; MARTINELLI, N.M.; MACCAGNAN, D.H.B. Dispersão de Fidicinoides sp. (Hemiptera: Cicadidae) em cafeeiro. Científica, v.34, p.263-268, 2006.

SANBORN, A.F. New records of Brazilian cicadas including the description of a new species (Hemiptera: Cicadoidea, Cicadidae). Neotropical Entomology, v.37, p.685-690, 2008.

SANBORN, A.F. A new species of Orellana Distant from Brazil (Hemiptera: Cicadoidea: Cicadidade). Proceedings of the Entomological Society of Washington, v.113, p.377-384, 2011a.

SANBORN, A.F. Checklist of the cicadas of French Guiana including new records and the description of nine new species (Insecta, Hemiptera, Cicadoidea, Cicadidae). Zoosystema, v.33, p.377-418, 2011 b.

SANBORN, A.F.; HEATH, J.E.; PHILLIPS, P.K.; HEATH, M.S.; NORIEGA, F.G. Thermal adaptation and diversity in tropical ecosystems: evidence from cicadas (Hemiptera, Cicadidae). PLoS ONE, v.6, p.1-12, 2011.

SANTOS, R.S.; MARTINELLI, N.M. Primeiro registro de Fidicinoides picea (Walker, 1850) e Fidicinoides poulaini Boulard \& Martinelli, 1996 no Brasil. Ciência Rural, v. 39, p.559-562, 2009a.

SANTOS, R.S.; MARTINELLI, N.M. Descriçâo de novas espécies de Fidicinoides Boulard \& Martinelli, 1996 (Hemiptera: Cicadidae) de ocorrência no Brasil. Neotropical Entomology, v.38, p.638-642, 2009b.

SANTOS, R.S.; MARTINELLI, N.M.; MACCAGNAN, D.H.B.; RIBEIRO, R.; SANBORN, A.F. Description of new cicada species associated with the coffee plant and an identification key for the species of Fidicinoides (Hemiptera: Cicadidae) from Brazil. Zootaxa, v.2602, p.48-56, 2010.

ZANUNCIO, J.C.; PEREIRA, F.F.; ZANUNCIO, T.V.; MARTINELLI, N.M.; PINON, T.B.M.; GUIMARÃES, E,M. Occurrence of Quesada gigas on Schizolobium amazonicum trees in Maranhão and Pará States, Brazil. Pesquisa Agropecuária Brasileira, v.39, p.943-945, 2004. 